\title{
How to detect the rare BCR-ABL (e14a3) transcript: A case report and literature review
}

\author{
LIN-HUI HU, LIAN-FANG PU, DONG-DONG YANG, CUI ZHANG, HUI-PING WANG, \\ YANG-YANG DING, MAN-MAN LI, ZHI-MIN ZHAI and SHUDAO XIONG \\ Hematological Lab, Department of Hematology, \\ The Second Hospital of Anhui Medical University, Hefei, Anhui 230601, P.R. China
}

Received January 27, 2016; Accepted December 20, 2016

DOI: $10.3892 / \mathrm{ol} .2017 .6847$

\begin{abstract}
The Philadelphia (Ph; BCR-ABL) chromosome originates from a translocation event between chromosomes 9 and 22, and results in the BCR-ABL fusion gene. In chronic myelogenous leukemia (CML), the BCR-ABL gene is mainly coded for by a major breakpoint cluster region (M-bcr, e13a2 and e14a2). However, in some patients, BCR-ABL genes are encoded by a minor (m)-bcr, ela2, and a micro $(\mu)$-bcr region, e19a2. These transcripts revealed a different clinical course. The present study described a CML patient whose cytogenetics and FISH analyses of bone marrow revealed a karyotype of 46, XY t(9,22) (q34;q11), while the commercial kits of quantitative PCR (qPCR) failed to detect the BCR-ABL fusion gene. Further multiplex Reverse transcription-PCR (RT-PCR) and sequencing analyses identified a rare e14a3 (b3a3) fusion transcript.
\end{abstract}

\section{Introduction}

A translocation event that occurs between chromosomes 9 and 22, $\mathrm{t}(9 ; 22)(\mathrm{q} 34 ; \mathrm{q} 11)$, results in the Philadelphia (Ph) chromosome, and consequently the BCR-ABL fusion gene, which is transcribed and translated into a hybrid protein (1). This fusion gene comprises almost the entire coding region of the Abelson (ABL) gene on chromosome 9, and the changeable coding region of the breakpoint cluster region (BCR) gene on chromosome 22 (2). In chronic myelogenous leukemia (CML), the breakpoint region lies between exons 12 and 16, and is termed the major breakpoint cluster region (M-bcr). The translocation events involving the M-bcr transcripts e13a2 (b2a2) and e14a2 (b3a2) encode a $210 \mathrm{kDa}$ protein termed P210. Translocations that occur within the minor(m)-bcr region retain only the first exon of the BCR gene transcripts, ela2, which yields a smaller P190

Correspondence to: $\mathrm{Dr}$ Shudao Xiong, Hematological Lab, Department of Hematology, The Second Hospital of Anhui Medical University, 678 Furong Road, Shushan, Hefei, Anhui 230601, P.R. China

E-mail: xshdao@163.com

Key words: BCR-ABL, e14a3, chronic myelogenous leukemia gene product. A rare $\mathrm{P} 230$ fusion protein, with a micro $(\mu)$-bcr breakpoint between exons 19 and 20, has also been described in a number of variant cases (3). In addition, some rare breakpoints have also been sporadically reported, which may be grouped into 4 categories: The BCR breakpoints originating within introns that lie outside the M-bcr, m-bcr, or $\mu$-bcr fused to ABL a2; the BCR breakpoints occurring within exons fused to ABL a2; the typical BCR breakpoints M-bcr, m-bcr, or $\mu$-bcr fused to ABL breakpoints located downstream of a2; and the transcripts containing intervening sequences between BCR and ABL a2 (4).

The National Comprehensive Cancer Network (NCCN) practice guidelines recommend that cytogenetics, fluorescence in situ hybridization (FISH) and quantitative polymerase chain reaction (qPCR) are used for the diagnosis of the chronic phase of adult CML (5). Cytogenetics and FISH detect the translocation event, yet cannot determinate the breakpoints on the chromosome, whereas qPCR is able to detect these breakpoints. However, special primers or probes are required when qPCR is used to detect these breakpoints on the chromosome. Numerous qPCR probes and primers have been designed to detect the common BCR-ABL transcript, but false negative results may present in patients with atypical transcripts.

The present study reported the data from a CML patient whose cytogenetics and FISH analyses of bone marrow revealed a karyotype of 46, XY, t(9,22)(q34;q11); however, qPCR failed to detect the BCR-ABL fusion gene. The PCR products by multiplex RT-PCR assay identified a band of $\sim 300 \mathrm{bp}$, which were subsequently confirmed as the BCR-ABL e14a3 transcript through sequence analysis. In addition, the biological role of the rare fusion event was reviewed, and the ability of qPCR assays to detect this rare transcript was discussed in the present study.

\section{Case report}

A 40 year-old male, whose white blood cell (WBC) count had been elevated for several days, was referred to The Second Hospital of Anhui Medical University, Hefei, China, in November 2015 for evaluation of his markedly high leukocyte level of $46.42 \times 10^{9} / 1$. No fever, hidrosis and musculoskeletal pain were found in the course of his physical examination. The peripheral blood test revealed hemoglobin (HB) levels of $132 \mathrm{~g} / 1$, a platelet count (PLT) of 275x10 $/ 1$ (67.6\% neutrophils, $21.3 \%$ 
lymphocytes and $9.2 \%$ basophil cells). The bone marrow examination revealed marked hyper-cellularity, with an increased number of megakaryocytes and evidence of myeloid hyperplasia and basophilia. The chromosomal analysis presented a karyotype of $46, \mathrm{XY}, \mathrm{t}(9 ; 22)(\mathrm{q} 34 ; \mathrm{q} 11)$. The FISH analysis produced a positive result for the BCR-ABL translocation. Based on these findings the patient was diagnosed with CML, and was treated with $400 \mathrm{mg} /$ day imatinib. The patient appeared to respond well to this treatment, as demonstrated in Fig. 1.

\section{Materials and methods}

Cytogenetic analysis was performed on bone marrow aspirates according to the standard laboratory protocol. A total of twenty G-banded metaphases and two karyotypes were evaluated. LSI BCR/ABL dual color, dual fusion translocation probe set (Abbott Molecular Inc., Des Plaines, IL, USA) was used to performed FISH analysis, following the protocol of the manufacturer. Total RNA was extracted from EDTA-anticoagulated bone marrow samples using an UN1Q-10 Column RNA Isolation kit (Sangon Biotech Co., Ltd., Shanghai, China), following the protocol of the manufacturer. A multiplex RT-PCR was carried out as described previously (6). A qPCR assay was performed to detect the BCR-ABL fusion gene, using a quantitative fluorescent RNA detection kit (Shanghai Shenyou Bio Technology Co., Ltd., Shanghai, China). The human leukemia sup-b15 (e1a2), nalm-6 (BCR-ABL negative) cell lines, were purchased from JENNIO Biological Technology (Guangzhou, China), and BV173 (e13a2) cell line, was provided by Dr Mengqing Gao (Shanghai Jiao Tong University School of Medicine, Shanghai, China), were used as controls. Multiplex RT-PCR products were sequenced by Sangon Biotech Co., Ltd. The sequence of Multiplex RT-PCR product was clarified by BLASTN (National Center for Biotechnology Information; blast.ncbi.nlm.nih.gov/Blast.cgi). All the data of patient were collected according to the approval of the institutional review board and informed consent was obtained.

\section{Results}

The chromosomal analysis of the patient presented a karyotype of $46, \mathrm{XY}, \mathrm{t}(9 ; 22)(\mathrm{q} 34 ; \mathrm{q} 11)$ in all 20 metaphases examined, as demonstrated in Fig. 2. FISH analysis detected the BCR-ABL translocation, as displayed in Fig. 3. Commercial kits of qPCR failed to detect the BCR-ABL fusion gene. The multiplex RT-PCR performed on the RNA did not detect the classic BCR-ABL P210 transcript in the cells obtained from the bone marrow of the patient, or the sup-b15 or nalm- 6 cell lines, but was detected in the BV173 cell line with the e13a2 transcript. However, the multiplex RT-PCR product of the patient demonstrated a band measuring $\sim 300$ bp in $2 \%$ agarose gel, as demonstrated in Fig. 4. The multiplex RT-PCR product was sequenced by Sangon Biotech Co., Ltd., and confirmed the presence of the e14a3 BCR-ABL transcript (b3a3) by BLASTN analysis, which is showed in Fig. 5.

\section{Discussion}

In patients with CML with the Ph chromosomal abnormality, almost all fusion transcripts are e13a2 and e14a2 (M-bcr),

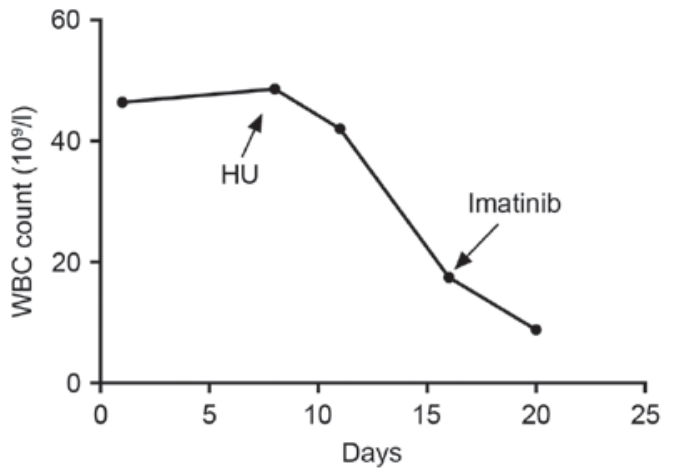

Figure 1. WBC count of the patient. At day 8, the patient was treated with $\mathrm{HU}$. At day 16, the patient was treated with imatinib. Arrows indicate the time-point of treatment initiation in the patient. WBC, white blood cell; HU, hydroxyurea.

which produce a $210 \mathrm{kDa}$ protein when transcribed (7). In rare variant patients, e1a2 (m-bcr) and e19a2 ( $\mu$-bcr) transcripts are found. The breakpoint of these transcripts usually occurs in ABL exon2, but occasionally arises in ABL exon3. The protein transcribed by exon 2 contains tyrosine kinase (SH1), SH2 and SH3 domains. In comparison with the proteins transcripted by exon2, the structure of the protein transcribed by exon3 lacked two-thirds of the $\mathrm{N}$-terminal in the $\mathrm{SH} 3$ domain. A previous study determined that in vivo, $\mathrm{SH} 3$ domains negatively regulate the kinase domain (SH1) and activate the Signal transducer and activator of transcription 5-signaling pathway, which is an essential leukemogenic factor (8). Furthermore, mouse models have demonstrated lower tissue invasiveness and longer leukemic cell cycles when BCR-ABL mutants lack the SH3 domain (9). Consequently, it is expected that patients who exhibit BCR-ABL without exon2 may present with a different clinical course.

To our knowledge, there have been 14 reports, including the patient of the present study, of patients with the BCR-ABL e14a3 transcripts $(5,10-15)$ (Table I). The median age of these patients is 44.5 years old: Patients with BCR-ABL e14a3 transcripts appear to be younger than patients with the common transcript at the time of diagnosis. Notably, 12 patients were male, suggesting that the BCR-ABL e14a3 transcript is more common in males. In addition, these 14 patients exhibited a less aggressive form of the disease and lower WBC counts, ranging from $9-300 \times 10^{9} / 1$. It was also noted that the deletion of exon2 did not affect the curative effects of imatinib, and 7 patients appeared to respond well to treatment with this drug $(5,10)$. The patient in the present study also appeared to have a good response to imatinib. Combining these data with previous studies $(5,10,16)$, patients with the BCR-ABL e14a3 transcript may expect a good clinical course.

The present study observed that the multiplex RT-PCR produced negative results for e13a2 and e14a2, but exhibited a band measuring $\sim 300 \mathrm{bp}$ in the agarose gel. qPCR analysis to detect the BCR-ABL fusion gene was then performed, but this failed to detect the BCR-ABL fusion gene. However, the $\mathrm{Ph}$ chromosome was detected by cytogenetics and FISH analyses. To additionally investigate the discordant result, the multiplex RT-PCR products were sequenced and analyzed by BLASTN, which confirmed the atypical transcript e14a 3 in the patient 
Table I. Summary of CML patients and the respective BCR-ABL e14a3 transcripts.

\begin{tabular}{|c|c|c|c|c|c|c|c|c|c|}
\hline Case no. & $\begin{array}{l}\text { Age/ } \\
\text { gender }\end{array}$ & $\begin{array}{l}\text { Phase } \\
\text { of CML }\end{array}$ & $\begin{array}{c}\text { WBC } \\
\text { count } \\
\left(\times 10^{9} / 1\right)\end{array}$ & $\begin{array}{c}\text { Chromosome } \\
\text { karyotype }\end{array}$ & $\begin{array}{l}\text { BCR-ABL } \\
\text { transcript }\end{array}$ & $\begin{array}{l}\text { Clinical } \\
\text { outcome }\end{array}$ & $\begin{array}{l}\text { Duration of } \\
\text { follow-up } \\
\text { (months) }\end{array}$ & Splenomegaly & References \\
\hline 1 & $39 / \mathrm{F}$ & $\mathrm{CP}$ & 9 & $\mathrm{~T}(9,22) 13 \mathrm{Q}$ & E14A3 & Alive & Unknown & NA & (10) \\
\hline 2 & 19/M & $\mathrm{CP}$ & 42 & $\mathrm{~T}(9,22)$ & E14A3 & Dead & 34 & NA & (11) \\
\hline 3 & 23/M & $\mathrm{CP}$ & 95.8 & $\mathrm{~T}(9,22)$ & E14A3 & Alive & 60 & $\mathrm{NO}$ & (12) \\
\hline 4 & $51 / \mathrm{M}$ & $\mathrm{CP}$ & NA & $\mathrm{T}(9,22)$ & E14A3 & Alive & 36 & $\mathrm{NO}$ & (16) \\
\hline 5 & 69/M & $\mathrm{CP}$ & 18 & $\mathrm{~T}(4,9,22)$ & E14A3 & Alive & 126 & $\mathrm{NO}$ & (16) \\
\hline 6 & 69/M & $\mathrm{CP}$ & 29 & $\mathrm{~T}(9,22)$ & E14A3 & Alive & Unknown & $\mathrm{NO}$ & (13) \\
\hline 7 & $81 / \mathrm{M}$ & $\mathrm{CP}$ & 28 & $\mathrm{~T}(9,22)$ & E14A3 & Alive & Unknown & NA & (5) \\
\hline 8 & $30 / \mathrm{M}$ & $\mathrm{CP}$ & NA & $\mathrm{T}(9,22)$ & E14A3 & Alive & Unknown & $\mathrm{NO}$ & (14) \\
\hline 9 & $52 / \mathrm{M}$ & $\mathrm{CP}$ & 229 & $\mathrm{~T}(9,22)$ & E14A3 & Alive & 112 & YES & (9) \\
\hline 10 & $41 / \mathrm{M}$ & AP & 26 & $\mathrm{~T}(9,22)$ & E14A3 & Alive & 66 & YES & (9) \\
\hline 11 & 41/M & $\mathrm{CP}$ & 115 & $\mathrm{~T}(9,22)$ & E14A3 & Alive & 101 & YES & (9) \\
\hline 12 & $48 / \mathrm{F}$ & $\mathrm{CP}$ & 300 & $\mathrm{~T}(9,22)$ & E14A3 & Dead & 53 & YES & (9) \\
\hline 13 & 48/M & $\mathrm{CP}$ & 98.2 & $\mathrm{~T}(9,22)$ & E14A3 & Alive & 30 & YES & (9) \\
\hline 14 & $40 / \mathrm{M}$ & $\mathrm{CP}$ & 46 & $\mathrm{~T}(9,22)$ & E14A3 & Alive & 2 & NO & $\begin{array}{l}\text { Present } \\
\text { study }\end{array}$ \\
\hline
\end{tabular}

M, male; F, female; CP, chronic phase, CML; AP, accelerated phase; CML, chronic myeloid leukemia; WBC, white blood cell; BCR-ABL, Philadelphia chromosome.

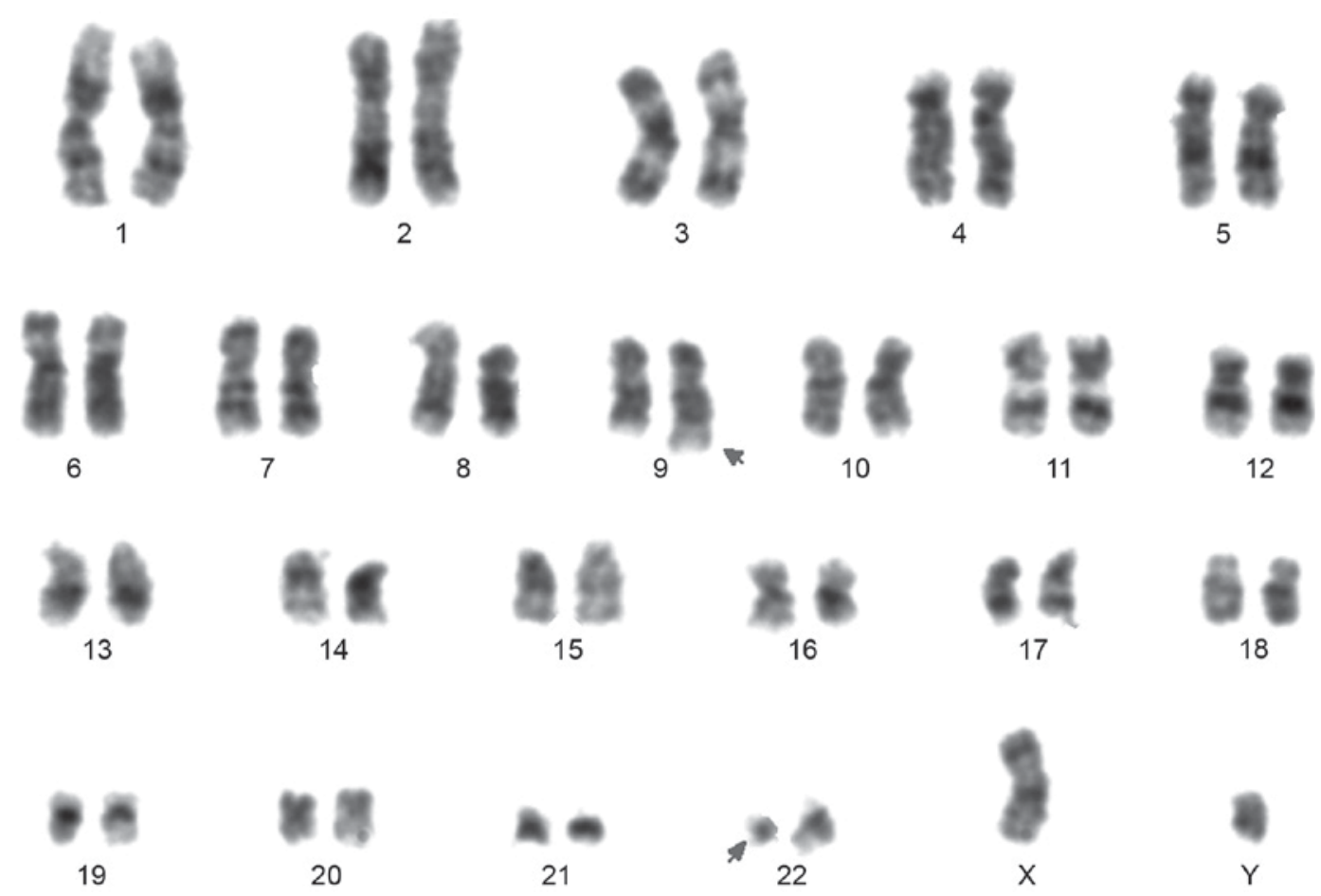

Figure 2. Giemsa-banding karyogram of a bone marrow sample from the patient: 46, XY, t(9:22)(q34;q11.2). Arrows indicate the derivative chromosomes 9 and 22.

of the present study. Although qPCR failed to detect this transcript, the multiplex RT-PCR assay previously described succeeded in detecting the BCR-ABL e14a3 transcript. Based on these findings, the present study suggested that multiplex RT-PCR assay reported by Pallisgaard et al (6) may provide evidence that PCR products amplified by R6B group presents a band with $\sim 300 \mathrm{bp}$, and associated with BCR-ABL e14a3 transcript.

The NCCN practice guidelines recommend that cytogenetics, FISH and qPCR be performed to diagnose the chronic phase of adult CML. In the present study, cytogenetics and FISH were successful in detecting the BCR-ABL e14a3 


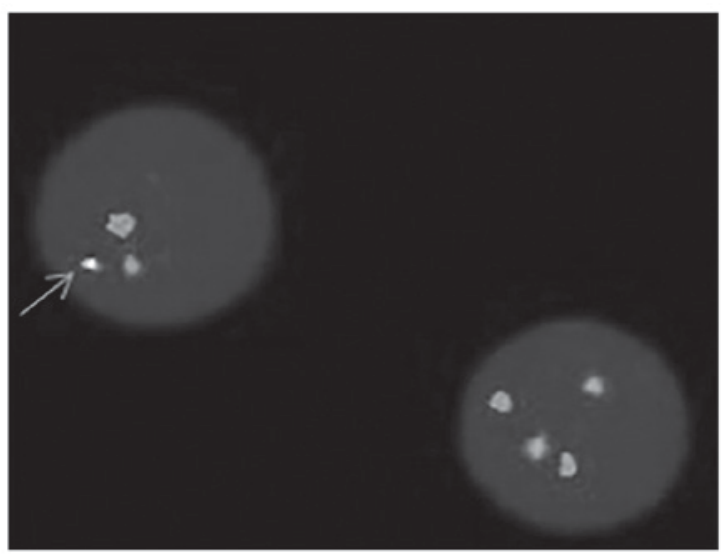

Figure 3. One fusion signal obtained via FISH analysis, indicative of the presence of $1 \mathrm{Ph}$ chromosome. Arrow indicates the $\mathrm{Ph}$ chromosomes.

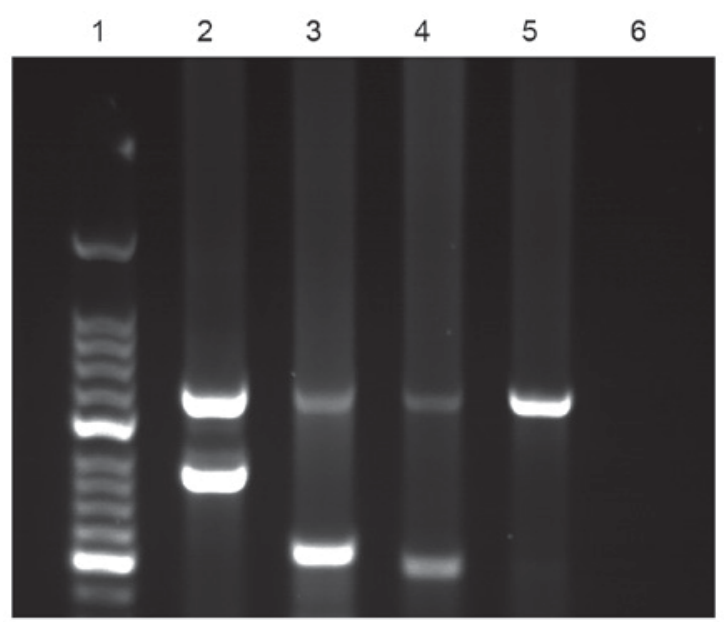

Figure 4. Multiplex RT-PCR products in 2\% agarose gel. Lane 1: Size marker 50 bp ladder. Lane 2: Multiplex RT-PCR product of the BCR-ABL transcript of the BV173 cell line (e13a2), 472 bp. Lane 3: Multiplex RT-PCR product of sup-b15 cell line (e1a2), 320 bp. Lanes 4: Multiplex RT-PCR product of the BCR-ABL transcript of the patient (e14a3). Lane 5: Multiplex RT-PCR product of nalm-6 cell line (BCR-ABL negative). Lane 6: Blank. RT-PCR, reverse transcription-polymerase chain reaction; BCR-ABL, Philadelphia chromosome.

transcript in the patient. However, the qPCR failed, due to a lack of specific primers, which makes repeating the qPCR every 3-6 months, as recommend by the NCCN, useless. Presently, some commercial qPCR kits are able to detect the e14a3 and e13a3 transcripts using the reverse primer located in exon 4 of ABL, such as Roche Holding AG, Basel, Switzerland and Applied Biosystems, Thermo Fisher Scientific Co., Waltham, MA, USA (5), while special primers that are able to detect the e14a3 transcript have also been designed (17). However, these assays fail to distinguish the type of BCR-ABL transcript present. Different BCR-ABL transcripts result in different clinical courses (18), and the impact of the type of transcript on response and survival rate subsequent to initial treatment with different tyrosine kinase inhibitors demonstrates different outcomes (19). True categorization of the BCR-ABL transcript by breakpoint is required in order to investigate and comprehend the different types of CML, and thus provides accurate guides for the treatment and prognosis of the disease.

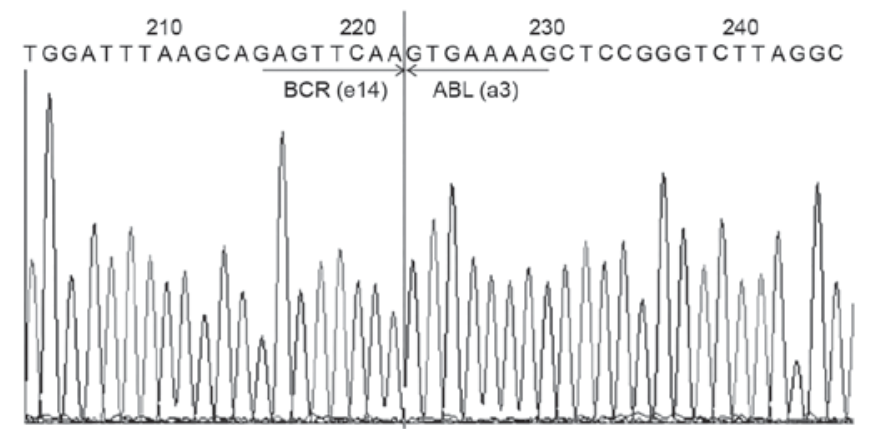

Figure 5. Sequencing result of the multiplex PCR product. Vertical line indicates the breakpoint and BCR-ABL junction of the fusion gene. PCR, polymerase chain reaction; BCR-ABL, Philadelphia chromosome.

In conclusion, a qPCR assay using commercial kits or primers designed by previous studies, which would detect the exon a3 transcripts, in order to estimate the disease progression of CML in a patient with the e14a3 transcript. Furthermore, in the future, multiplex RT-PCR assays may be applied to distinguish the type of BCR-ABL transcript present, with different transcripts demonstrating different sizes. Also, it is necessary to explore the possibility of a new qPCR assay that detects and distinguishes different BCR-ABL transcripts e13a2, e14a2, e13a3 and e14a3 respectively.

\section{References}

1. Rowley JD: Letter: A new consistent chromosomal abnormality in chronic myelogenous leukaemia identified by quinacrine fluorescence and Giemsa staining. Nature 243: 290-293, 1973.

2. de Klein A, van Kessel AG, Grosveld G, Bartram CR, Hagemeijer A, Bootsma D, Spurr NK, Heisterkamp N, Groffen J and Stephenson JR: A cellular oncogene is translocated to the Philadelphia chromosome in chronic myelocytic leukaemia. Nature 300: 765-767, 1982.

3. Bernt KM and Hunger SP: Current concepts in pediatric Philadelphia chromosome-positive acute lymphoblastic leukemia. Front Oncol 4: 54, 2014.

4. Barnes DJ and Melo JV: Cytogenetic and molecular genetic aspects of chronic myeloid leukaemia. Acta Haematol 108: 180-202, 2002.

5. Jinawath N, Norris-Kirby A, Smith BD, Gocke CD, Batista DA, Griffin CA and Murphy KM: A rare e14a3 (b3a3) BCR-ABL fusion transcript in chronic myeloid leukemia: Diagnostic challenges in clinical laboratory practice. J Mol Diagn 11: 359-363, 2009.

6. Pallisgaard N,Hokland P,Riish øj DC, Pedersen B and Jørgensen P: Multiplex reverse transcription-polymerase chain reaction for simultaneous screening of 29 translocations and chromosomal aberrations in acute leukemia. Blood 92: 574-588, 1998.

7. Bernards A, Rubin CM, Westbrook CA, Paskind M and Baltimore D: The first intron in the human c-abl gene is at least 200 kilobases long and is a target for translocations in chronic myelogenous leukemia. Mol Cell Biol 7: 3231-3236, 1987.

8. Nieborowska-Skorska M, Wasik MA, Slupianek A, Salomoni P, Kitamura T, Calabretta B and Skorski T: Signal transducer and activator of transcription (STAT) 5 activation by BCR/ABL is dependent on intact Src homology (SH)3 and SH2 domains of BCR/ABL and is required for leukemogenesis. J Exp Med 189: 1229-1242, 1999

9. Skorski T, Nieborowska-Skorska M, Wlodarski P, Wasik M, Trotta R, Kanakaraj P, Salomoni P, Antonyak M, Martinez R, Majewski M, et al: The SH3 domain contributes to BCR/ABL-dependent leukemogenesis in vivo: Role in adhesion, invasion, and homing. Blood 91: 406-418, 1998.

10. Iwata S, Mizutani S, Nakazawa S and Yata J: Heterogeneity of the breakpoint in the ABL gene in cases with BCR/ABL transcript lacking ABL exon a2. Leukemia 8: 1696-1702, 1994. 
11. Polák J, Zemanová Z, Michalová K, Klamová H, Cermák J and Haskovec C: A new case of chronic myeloid leukemia (CML) in myeloid blast crisis with an atypical (b3/a3) junction of the BCR/ABL gene. Leukemia 12: 250, 1998.

12. Amabile M, Martinelli G, Terragna C, Montefusco V, Tabilio A and Tura S: An atypical (b3/a3) junction of the bcr/abl gene lacking abl exon a 2 in a patient with chronic myeloid leukemia Haematologica 84: 573-575, 1999.

13. Paz-Y-Miño C, Arévalo M and Leone PE: B3/A3 rearrangement in a patient with chronic myeloid leukemia. Leuk Lymphoma 44: 375-376, 2003.

14. Vaniawala S, Acharya A, Parekh $\mathrm{H}$ and Mukhopadhyaya PN: Rare e14a3 (b3a3) BCR-ABL fusion in chronic myeloid leukemia in India: The threats and challenges in monitoring minimal residual disease (MRD). Anal Cell Pathol (Amst) 36: 85-92, 2013.

15. Xiaomin G, Zhang Y, Pan J, Qiu H, Cen J, Xue Y, Chen S, Shen H, Yao L, Zhang J, et al: Chronic myeloid leukemia with e14a3 BCR-ABL transcript: Analysis of characteristics and prognostic significance. Leuk Lymphoma 56: 3343-3347, 2015.
16. Tiribelli M, Tonso A, Ferrro D, Parzilae A, Cambrin GR, Scaravaglio P, Cilloni D, Gottardi E and Saglio G: Lack of SH3 domain does not imply a more severe clinical course in $\mathrm{Ph}+$ chronic myeloid leukemia patients. Blood 95: 4019-4020, 2000.

17. Kreuzer KA, Lass U, Bohn A, Landt O and Schmidt CA LightCycler technology for the quantitation of bcr/abl fusion transcripts. Cancer Res 59: 3171-3144, 1999.

18. Hanfstein B, Lauseker M, Hehlmann R, Saussele S, Erben P, Dietz C, Fabarius A, Proetel U, Schnittger S, Haferlach C, et al: Distinct characteristics of e13a2 versus e14a2 BCR-ABL1 driven chronic myeloid leukemia under first-line therapy with imatinib. Haematologica 99: 1441-1457, 2014.

19. Jain P, Kantarjian H, Patel KP, Gonzalez GN, Luthra R, Kanagal Shamanna R, Sasaki K, Jabbour E, Romo CG, Kadia TM, et al: Impact of BCR-ABL transcript type on response and survival in patients with chronic-phase chronic myeloid leukemia treated with tyrosine kinase inhibitors. Blood 127: 1269-1275, 2016. 